\title{
EDUCAÇÃO EM SAÚDE: PLANEJ AMENTO E EXECUÇÃO DA ALTA EM UMA UNIDADE DE TERAPIA INTENSIVA NEONATAL
}

\author{
Education in health: planning and implementation of the discharge in a neonatal intensive \\ care unit
}

\section{Educación en salud: planificación y ej ecución de alta en una unidad de cuidados intensivos neonatales}

Elisabeta Albertina Nietsche
Janilene Camara Bottega

\section{RESUMO}

Objetivou-se compreender a percepção dos profissionais de saúde e pais em relação ao planejamento e efetivação da alta do recém-nascido da Unidade de Terapia Intensiva Neonatal. Trata-se de uma pesquisa exploratório-descritiva, com abordagem qualitativa, cuja coleta de dados foi realizada por meio de entrevista semiestruturada em 2011. Os participantes da pesquisa foram oito profissionais da saúde e treze pais de recém-nascidos. Para análise dos dados utilizou-se a Análise de Conteúdo. Os resultados apontam que a alta hospitalar dos neonatos é focada no restabelecimento das condições fisiológicas; as orientações de cuidado ao recém-nascido são passadas aos pais pelo enfermeiro de forma rápida e acontecem apenas no momento da alta. Concluiu-se que é necessária ampliação das estratégias de educação em saúde no processo de alta hospitalar, para os pais de neonatos, pela equipe de saúde em sua rotina de trabalho, visando melhor planejamento e continuidade do cuidado prestado.

Palavras-chave: Alta do paciente. Recém-nascido. Educação em saúde. Enfermagem.

\section{Abstract}

It was aim to understand the perception of professionals and parents in relation to the planning and realization of the discharge of the newborn in Neonatal Intensive Care Unit. It is an exploratorydescriptive research with qualitative approach which data collection was obtained through a semi-structured interview in 2011. The participants were eight health professionals and thir teen parents of newborn. For the analysis of the data it was used Content Analysis. The results indicate that the hospital discharge of newborns is focused in the reestablishment of physiological conditions; the care guidelines for newborn are passed to the parents by the nurse, in a fast way and it happens only in the discharge time. It was concluded that it is necessary the expansion of health education strategies in the discharge process, for parents of newborns, for the health team in your everyday work, for better planning and continuity of the care provided.

Keywords: Patient discharge. Newborn. Health education. Nursing.

\section{Resumen}

Se objetivó comprender la percepción de los profesionales de salud y de los padres sobre la planificación y ejecución del alta al recién nacido en una Unidad de Cuidados Intensivos Neonatales. Investigación exploratoria-descriptiva, con abordaje cualitativo, cuya recolección de datos fue a través de entrevistas semi-estructuradas, en 2011. Los participantes fueron ocho profesionales de salud y trece padres de recién nacidos. Para análisis de datos se utilizó el Análisis de Contenido. Los resultados indican que el proceso de darle alta hospitalario al recién nacido es centrado en la restauración de condiciones fisiológicas; las orientaciones de atención al recién nacido son transmitidas a los padres por el enfermero, rápidamente y sólo en el alta. Se concluyó que es necesaria la expansión de las estrategias de educación en salud en el proceso del alta para los padres de recién nacidos, por el equipo de salud en su trabajo diario, para mejor planificación y continuidad de la atención prestada.

Palabras-Clave: Alta del paciente. Recién nacido. Educación en Salud. Enfermería.

\footnotetext{
${ }_{1}^{1}$ Doutora em Enfermagem pela Universidade Federal de Santa Catarina. Docente do Departamento e do Programa de Pós-Graduação em Enfermagem da Universidade Federal de Santa Maria (PPGEnf./UFSM). Coordenadora do Grupo de Estudos e Pesquisa (GEPES/UFSM/CNPQ). Santa Maria-RS. Brasil. Email: enietsch@terra.com.br; ${ }^{2}$ Graduanda do $8^{\circ}$ semestre do Curso de Enfermagem da UFSM. Integrante do GEPES/UFSM/CNPQ. Santa Maria-RS. Brasil. E-mail: aline-dallanora@hotmail.com; ${ }^{3}$ Enfermeira. Mestranda e Bolsista CAPES pelo PPGEnf./UFSM. Integrante do GEPES/UFSM/CNPQ. Santa Maria-RS. Brasil. E-mail: grlmarcia@yahoo.com.br; ${ }^{4}$ Acadêmica de Enfermagem do $7{ }^{\circ}$ semestre de Graduação da UFSM. Integrante do GEPES/UFSM/ CNPQ. Santa Maria-RS. Brasil. E-mail: janicamega@yahoo.com.br; ${ }^{5}$ Doutora em Enfermagem pela Universidade Federal do Rio de Janeiro. Estágio doutoral junto a Culture and Mental Health Research Unit (Mc Gill University - Montreal/Canadá). Coordenadora do Curso de Graduação em Enfermagem do CCS/UFSM. Docente do Departamento e do PPGEnf./UFSM. Membro do Grupo de Pesquisa Cuidado à Saúde de Pessoas, Família e Sociedade - PEFAS. Santa MariaRS. Brasil. E-mail: elianeves03@gmail.com; ${ }^{6}$ Enfermeira da Unidade de Terapia Intensiva Neonatal do Hospital Universitário de Santa Maria. Santa MariaRS. Brasil. E-mail: veralucia@yahoo.com.br
} 


\section{INTRODUÇÃO}

Na primeira metade do século XX muitas transformações ocorreram no cuidado à saúde da criança, principalmente no atendimento aos recém-nascidos (RN) ${ }^{1}$. No Brasil, o significativo desenvolvimento tecnológico, a partir da última década, possibilitou otimizar a assistência intensiva ofertada aos RN, por meio dos serviços da Unidade de Terapia Intensiva Neonatal (UTIN) ${ }^{1}$.

0 período neonatal compreende os 28 primeiros dias de vida da criança e caracteriza-se pela ocorrência de inúmeras adaptações anatômicas, fisiológicas e, sobretudo, familiares². Então, quando um RN apresenta condições clínicas de risco, como: prematuridade, malformações nos sistemas cardiovascular, neurológico, gastrintestinal, patologias respiratórias, entre outras, ele é internado na UTIN³.

Nesta perspectiva, quando o neonato apresenta alguma patologia que ameace sua vida, os pais percebem-se diante de uma experiência desgastante e desafiadora, que resultará em sensações múltiplas, sendo a primeira delas a preocupação com a sobrevivência dessa criança ${ }^{4}$. Outro agravante é a necessidade de hospitalização do $\mathrm{RN}$, a qual provoca diversas alterações na dinâmica dos pais e promove sua inserção em um ambiente repleto de rotinas, equipamentos, profissionais e procedimentos desconhecidos ${ }^{5}$.

Diante disso, a hospitalização do RN pode gerar medo, cansaço físico e mental àqueles pais que aguardam ansiosos a estabilidade clínica e, também, o ganho de peso do bebê, condições essas indispensáveis para a alta hospitalar. Então, é extremamente necessário que os membros da equipe de saúde compreendam as reações dos pais, a fim de evitar sentimentos negativos neste núcleo, pois uma de suas ações é proporcionar apoio nos momentos de crise ${ }^{6}$.

Por outro lado, apesar da atenção neonatal experimentar, atualmente, importantes avanços com a incorporação de novas tecnologias, ainda persiste a dificuldade na implementação de ações de educação em saúde ${ }^{a}$ que garantam a continuidade da assistência ao RN no domicílio, provocando fragmentação na atenção à saúde ${ }^{7}$.

Corroborando essa questão, durante o período de internação, com frequência, o RN desenvolve patologias associadas aos procedimentos realizados em prol de sua sobrevivência, o que, muitas vezes, repercute em seu desenvolvimento futuro. Sendo assim, não basta oferecer acompanhamento rigoroso apenas durante sua permanência na unidade intensiva, mas também é fundamental acompanhálo na alta para unidades de cuidado intermediário, baixa complexidade e domicílio8.

Com referência à alta da UTIN, esta é considerada algo crítico, que exerce grande influência na integralidade e continuidade do cuidado, pois alguns pais apresentam dúvidas e inseguranças em relação aos cuidados básicos com o(a) filho(a) e quanto à possibilidade de necessitarem, temporária ou permanentemente, de cuidados especiais e de suporte tecnológico domiciliar ${ }^{6}$.

Partindo desse contexto, o planejamento do processo de alta hospitalar pelos profissionais de saúde e 0 acesso às informações sobre a continuidade da assistência no domicílio pelos pais é determinante à qualidade do cuidado prestado ao RN. Entretanto, as ações de educação em saúde nesse processo geralmente são feitas às vésperas do bebê ir para casa, quando acontecem, gerando ansiedade e dúvidas ${ }^{6}$.

Na vivência de acadêmicos da UTIN de um hospitalescola adquire-se afinidade pelo ambiente de terapia intensiva, pelo convívio com os RN e sua família, bem como o aprendizado sobre a complexidade da assistência sustentada nos princípios da integralidade a essa clientela. Isso permite perceber que se não houver preocupação com o preparo dos pais para cuidar dessa criança no local onde ela retornará, com certeza os tratamentos hospitalares não serão efetivos, mesmo com a utilização de tecnologias avançadas.

Dessa forma, o planejamento da alta hospitalar tem o papel de desenvolver o senso de responsabilidade nos pais para 0 atendimento do RN, ensinando sobre o modo mais adequado de prestar cuidados e responder às necessidades do seu(sua) ûlho(a), diminuindo o estresse, evitando readmissões e buscando recursos disponíveis na comunidade para 0 atendimento após a alta.

Esse estudo poderá contribuir na inserção de ações educativas tanto no planejamento quanto na execução da alta hospitalar do RN em unidade intensiva, pautadas na integralidade e continuidade da assistência, além de fomentar a reflexão sobre as práticas em saúde, a fim de dar visibilidade aos profissionais acerca da importância de fornecer aos pais acesso às informações, respeitando suas necessidades e proporcionando a conquista de sua autonomia na tomada de decisão quanto à saúde do RN.

Este artigo teve como objetivo compreender a percepção dos profissionais e dos pais em relação ao planejamento e efetivação da alta do RN da UTIN.

\section{METODOLOGIA}

Trata-se de um estudo do tipo exploratório-descritivo, com abordagem qualitativa. 0 cenário do estudo foi a UTIN de um hospital-escola de alta complexidade, alocado no interior do estado do Rio Grande do Sul, Brasil. Essa unidade oferece dezoito leitos classificados como de alto risco, divididos em: infectados e não infectados, e de risco intermediário. A equipe é composta por dez médicos, nove enfermeiros, trinta e nove técnicos e auxiliares de enfermagem, dois fisioterapeutas e uma psicóloga.

Os sujeitos da pesquisa compreenderam os profissionais da equipe multiprofissional de saúde da UTIN e os pais de RN internados nessa Unidade, no período da coleta de dados. Entretanto, os critérios de inclusão dos profissionais foram: estar atuando no mínimo há doze meses na unidade, 
considerando que nesse período é possível obter maior contato com paciente, família e rotinas da Unidade, bem como já haver participado em mais de uma alta hospitalar, pois a maioria dos RN permanece no mínimo por três meses internados. Destes profissionais foram escolhidos por conveniência (estar trabalhando na Unidade no período de coleta de dados): um médico, cinco enfermeiros, um psicólogo e um fisioterapeuta, totalizando oito profissionais.

Cabe ressaltar que, inicialmente, pretendia-se adotar cinco profissionais médicos como sujeitos da pesquisa; porém, após a inserção no campo, percebeu-se que havia apenas um médico fixo da UTIN, os demais médicos residentes e acadêmicos da medicina que permanecem pouco tempo na Unidade não atenderam aos critérios de inclusão dos sujeitos.

Em relação aos pais, foi adotado como critério de inclusão: ter RN internado na UTIN no mínimo há sete dias, por acreditar que este é o tempo mínimo necessário para que os pais estabeleçam contato com maior número de profissionais e se adaptem às rotinas do setor e à situação de internação do filho. Por isso, totalizaram-se treze pais. Assim, participaram da pesquisa 21 sujeitos.

A coleta de dados ocorreu em 2011, após apreciação do projeto pelo Comitê de Ética em Pesquisa da universidade, sob o número 0012.0.243.000.11. Utilizou-se a entrevista semiestruturada, a qual foi divida em dois instrumentos, um para os profissionais, com questões sobre: o processo; os procedimentos realizados; a articulação entre os serviços; sua influência e a participação dos profissionais na alta dos $\mathrm{RN}$ da UTIN, e o outro com questionamentos aos pais: quais as expectativas em relação à alta do bebê; as orientações e preparos recebidos para a alta do bebê.

0 encontro para entrevista com os sujeitos foi agendado previamente. Em relação aos profissionais, as entrevistas aconteceram em seu turno de serviço, na sala de Enfermagem, conforme sua disponibilidade, e os pais foram entrevistados após a visita ao RN, na sala de espera da Unidade. Nos encontros, foi entregue o Termo de Consentimento Livre e Esclarecido, em duas vias, uma para o participante e outra para o pesquisador, mediante a sua leitura para esclarecimentos referentes à pesquisa, concordância e assinatura.

0 processo de análise de dados, juntamente com a discussão e a interpretação destes, foi orientado pela técnica de Análise de Conteúdo de Bardin. Esse tipo de análise constituise em "um conjunto de técnicas de análise das comunicações muito empírico, que depende do tipo de fala a que se dedica e do tipo de interpretação que se pretende como objetivo" ":146. A análise de conteúdo divide-se em três polos cronológicos: pré-análise; exploração do material; tratamento dos resultados obtidos e interpretação?.

No primeiro momento da análise dos dados realizouse a comparação entre o conteúdo das entrevistas, agrupando quando houve semelhança. No segundo momento esse conteúdo foi articulado com o referencial teórico a fim de embasar, comparar e discutir teoricamente os resultados da investigação.

Ainda, assegurou-se a preservação da dignidade, respeito à autonomia e defesa da vulnerabilidade dos seres envolvidos na pesquisa, de acordo com a Resolução 196/96 do Conselho Nacional de Saúde. Garantiu-se também o anonimato dos participantes, mediante a adoção de pseudônimos, Membros da equipe (E1, E2, E3...) e Pais (P1, P2, P3....), para identificar seus depoimentos.

\section{RESULTADOS E DISCUSSÃO}

Procedendo ao processo de análise, discussão e interpretação dos dados, distribuiu-se o material obtido em categorias, a fim de buscar contornos do sistema de significações que, no seu conjunto, relacionassem a percepção dos profissionais e dos pais em relação ao planejamento e efetivação da alta do recém-nascido da UTIN.

Emergiram três categorias que representam o eixo em torno do qual o produto do estudo realizado se articula, a saber: percepção da equipe de saúde sobre a alta do recémnascido; influência do planejamento da alta do recém-nascido na continuidade do cuidado e percepção dos pais sobre as orientaç̃̃es de cuidado ao recém-nascido.

\section{Percepções da equipe de saúde sobre a alta do recém- nascido}

Em seus relatos os profissionais indicaram que a alta hospitalar do RN é uma atividade organizada, com etapas a serem seguidas, conforme descrito nas falas abaixo:

A alta é definida pelo médico e sua equipe, em
"round". São feitos todos os papéis burocráticos
pelos residentes e doutorandos que estão na sala
da alta. Após, eles passam para a secretária e para
a enfermeira que está no turno, que o bebê está de
alta. Geralmente o residente ou doutorando
conversa com a mãe, passa algumas informações;
e, antes de sair, sempre é o enfermeiro que passa
as informações de cuidado, dá os papéis, tira as
dúvidas (E5).

0 processo da alta é feito pela equipe médica. Depois, alguém da equipe de enfermagem, geralmente o enfermeiro, passa todas as instruções do que a mãe vai fazer, como: a marcação de consultas, as vacinas, o retorno, o cuidado em casa, e tira algumas dúvidas (E2).

A partir dessas falas percebe-se que o processo de alta dos RN é desencadeado, inicialmente, pelos médicos, os quais são responsáveis pela determinação de quando e qual RN terá alta, e os enfermeiros são responsáveis por passar aos pais as informações de cuidado na pós-alta. Diante disso, considera-se que o preparo para a alta hospitalar do RN não pode ser visto 
como um evento pontual e isolado, delegado a momentos prévios à saída do bebê, devendo ser vis-to e organizado continuamente, objetivando fornecer informações à família, capacitando-a para atuar em si-tuações críticas após sua saída do hospital ${ }^{10}$.

Um estudo realizado com mães que estavam acompanhando a criança no momento da alta para o domićílio constatou que o aviso de alta para o grupo estudado foi realizado 24 horas antes da sua efetivação, não havendo decisão conjunta ${ }^{6}$. Entretanto, o planejamento de alta envolve a formulação de um programa pela equipe de saúde, o paciente, a família e os órgãos externos apropriados para ir ao encontro das necessidades físicas e psicossociais do paciente após a alta, exigindo uma colaboração rigorosa da equipe de saúde ${ }^{6}$.

Por outro lado, a alta do RN é percebida por alguns profissionais como o momento em que há estabilidade clínica desses pacientes, como descrito a seguir:

Conforme ele [RN] vai ganhando peso ou melhorando o diagnóstico, ele passa por um processo, até que ganha alta (E1).

Primeiro o peso, cerca de $1900 \mathrm{~g}$ a $2 \mathrm{~kg}$. Quando a criança está mamando adequadamente todo 0 regime alimentar que é necessário para ela se sustentar, não está precisando de solução endovenosa, antibioticoterapia, oxigênio, está respirando adequadamente sem desconforto, conseguindo conservar a temperatura adequadamente em berço comum, pelo menos a 24hs, quando os pais tem condições de cuidar, quando a idade gestacional corrigida é de 34 semanas ou 35 no mínimo [...](E3).

O processo da alta é assim, quando a criança está respirando sem 02, tem o peso adequado, está mamando, não tem infecção nenhuma, hemograma normal (E6).

De acordo com as falas acima, o preparo da alta hospitalar dos RN se processa a partir do momento em que há o ganho de peso, a manutenção da temperatura corporal, controle e prevenção de infecções, capacidade de alimentação, respiração e idade gestacional adequados. Por outro lado, também énecessário haver o preparo dos pais, desde o momento em que eles vêm à unidade pela primeira vez, explicando os procedimentos realizados, estimulando sua participação no cuidado e favorecendo o vínculo, ao ensinar cuidados básicos de higiene, alimentação, manuseio do RN, supervisionando os procedimentos por eles executados, e, no momento da alta, no qual são reforçadas as orientações feitas e informadas sobre a importância dos mesmos ${ }^{10}$.

Entretanto, algumas ações empregadas no momento da alta dos RN, como as informações fornecidas aos pais, são percebidas como de difícil execução, como expressadas nas falas:

Eu vejo, assim, que é muita informação em pouco tempo. Até porque tem muitos pais que vem praticamente só na alta, ou vem poucas vezes. Então, não tem aquele acompanhamento diário com o bebê, e no dia da alta ela tem uma série de informações que são importantes e que é dada rapidamente. Imagino que fique difícil de assimilar todas essas informações que são importantes. Às vezes, a mãe está ansiosa para ir embora porque o transporte está esperando [...] e tu está passando as informações (E4).

Então, acho que o ideal, a meu ver, é que fosse dado algo por escrito, as informações que são passadas à mãe [...], para que ela na dúvida tivesse onde procurar (E3).

Segundo o relato desses profissionais é difíil fornecer orientações aos pais sobre os cuidados com o $\mathrm{RN}$ no processo da alta, pois muitos deles não participam do período de internação do filho. Porém, eles acreditam que o ideal seria passar as informações também por escrito. Em conformidade, um estudo ${ }^{11}$ desenvolvido com mães de RN prematuros que se encontravam na UTIN identificou, a partir do relato delas, a necessidade do fornecimento de material educativo com os cuidados a serem prestados diante das situações de risco para a criança.

Assim, torna-se fundamental a determinação de critérios para o planejamento da alta nas UTIN, reconhecendo que sejam padronizados, visto que não só confere maior coerência entre essas instituições, como também evita atrasos desnecessários ${ }^{12}$.

No tocante a estas orientações feitas aos pais, alguns profissionais confundem o significado de educação em saúde e educação em serviço, como abordado nas falas seguintes:

Todo o dia durante a internação a gente educa os pais, orienta, conforme as necessidades [...]. Às vezes, você dá o banho, você vai explicando, orientando. [A gente] dá orientação educacional aos pais, para quando o bebê der alta, terem condições de cuidar desse RN (E3).

Eu acho que a educação em serviço desses pais é fundamental. Se eles estão bem preparados, souberem manipular, cuidar, administrar a medicação, administrar o mamazinho corretamente [...]. É fundamental a educação em serviço que a gente faz para esses pais aqui (E6). 
Analisando a fala dos profissionais, perce-be-se que a educação em saúde é compreendida como orientação, transmissão de informações e retirada de dú-vidas, mas também confundida com educação em serviço. Em contrapartida, a educação em saúde, por sua vez, é um dos meios utilizados para a busca de uma melhor qualidade de vida, é considerada uma disciplina de ação, a qual visa atuar sobre o conhecimento das pessoas como forma de desenvolvimento da capacidade de intervenção na sua vida e no ambiente com o qual interagem ${ }^{12}$.

Já a educação em serviço caracteriza-se como um processo educativo a ser aplicado nas relações humanas do trabalho, no intuito de desenvolver capacidades cognitivas, psicomotoras e relacionais dos profissionais, assim como seu aperfeiçoamento diante da evolução científica e tecnológica ${ }^{13}$.

\section{Influência do planejamento da alta do recém-nascido na continuidade do cuidado}

As orientações que são repassadas aos pais sofrem repercussões na continuidade

do cuidado ao RN. Isso é abordado nas falas a seguir:

0 processo da alta influencia muito em casa, principalmente quando é o primeiro filho, que a mãe não tem experiência [...]. A gente orienta ela: a esgotar, a oferecer o seio, problemas eventuais que podem acontecer com a amamentação. Uma boa orientação desde a alta é fundamental, e não só no momento da alta [...], porque na verdade é uma educação que tem que ser contínua, para essa mãe, quando chega o momento da alta, ela ter as informações finais e estar preparada para cuidar desse bebê em casa, porque nem sempre elas têm experiência no cuidado com o bebê (E7).

Quando a criança está internada, a gente procura ir orientando porque não adianta você chegar dar todas as informações pra mãe na última hora, porque muita coisa ela vai esquecer e vai acabar não fazendo. Então, a gente observa como um processo lento e gradual [...], assim ela vai internalizando aquilo para dar continuidade a esse processo de cuidado em casa (E8).

A partir das falas desses profissionais, as orientações devem ser passadas aos pais de modo gradual e contínuo durante todo período de internação do RN, para auxiliar diretamente nos cuidados no domicílio. Então, a alta do neonato pode trazer implicações para a continuidade do cuidado, que se associam tanto a fatores relativos à assistência prestada ao mesmo, no cotidiano da internação, quanto a fatores relacionados ao planejamento da alta hospitalar.

Por consequência, as orientações não devem ser apenas assimiladas pelos pais, mas também compreendidas e incorporadas, sendo que o desenvolvimento de habilidades e aquisição de conhe-cimento deve partir dos pais por meio de estratégias estimuladoras desenvolvidas pela enfermagem ${ }^{14}$.

Em acréscimo, alguns profissionais apontam que 0 conhecimento dos pais acerca das patologias e das especificidades de alguns cuidados com seus filhos também influenciam na continuidade desse cuidado em casa, conforme as falas abaixo:

Eu acho que a mãe bem orientada [...], bem esclarecida sobre as patologias do bebê, como é que ela deve proceder, se o bebê tem alguma patologia é importante para ela ter essa continuidade. 0 que acontece é que muitos bebês retornam porque aspiraram o leite, precisando de aspiração nasal ou a mãe nem sabe o que fazer, onde ir [...] (E5).

Educando a mãe ela vai saber fazer os cuidados corretos, administrar a mamadeira, a higiene, a medicação; tem crianças que saem com medicação. Se ela está bem preparada ela vai dar continuidade a esse cuidado, e o bebê vai ganhar com isso (E6).

Esses profissionais destacam que orientar os pais sobre os cuidados ao RN no domićílio, com relação às patologias e sua interferência na amamentação, respiração, entre outros, é fundamental para dar continuidade aos cuidados, diminuindo a necessidade de o RN retornar a UTIN. Considera-se que o tratamento neonatal e o tempo de internação podem ter como consequência um elevado risco para morbidades, conferindo especial importância as informações destinadas ao suporte técnico e social adequado ${ }^{15}$.

Sob outro enfoque, a continuidade da assistência no domicílio também é influenciada pelo auxílio das demais especialidades oferecidas nos serviços da rede de saúde, como exposto a seguir pelos profissionais:

Quando ele [RN] vai dar alta, a gente tenta orientar o teste do pezinho, o teste da orelhinha [...], a consulta de puericultura que ele vai realizar depois no posto (E1).

A gente encaminha para o serviço da periferia [Unidade Básica] pra verificar como está indo o peso, como está indo o bebê, em relação à parte do seguimento pediátrico (E3).

Em geral, os pais até procuram a Unidade Básica, sempre oriento sobre a importância de dar continuidade. 0 grande diferencial é que aqui a gente atende todos os dias e em nenhum outro tipo de serviço eles vão ter esse atendimento. Aos 
pacientes que vão necessitar de um acompanhamento de fisioterapia, após a alta, oriento procurar um serviço de fisioterapia [...], e se a família for de fora [da região], eu oriento procurar algum serviço público em função da nossa demanda ser mais SUS [Sistema Único de Saúde] (E4).

A gente orienta para que o bebê continue tendo um atendimento com outro médico, com outro profissional, às vezes precisa de fisioterapeuta, de oftalmo, da fono [...] (E7).

Segundo esses relatos, a participação de outros profissionais e do apoio da própria rede de saúde é importante para dar continuidade ao cuidado prestado ao RN no domicílio. Assim, faz-se necessário aos profissionais da UTIN fazer o acompanhamento do bebê, visando reconhecer precocemente as condições clínicas que irão torná-lo dependente dos serviços de apoio à saúde, mas também conhecer a situação em que a família dessa criança está inserida para facilitar seu acesso.

Entretanto, a grande demanda é um problema nos serviços de saúde. Isso dificulta o acesso da população, em especial daquelas parcelas com menor poder aquisitivo, determinado tanto pela carência de serviços de transporte adequado quanto pela reduzida oferta de serviços ${ }^{16}$.

Outro aspecto que influencia na continuidade dos cuidados é a promoção do vínculo dos pais com o (a) filho (a), ainda no ambiente hospitalar. Isso é destacado nas seguintes falas:

Uma mãe bem vinculada, cuidadosa, vai ser uma mãe que vai estar atenta as necessidades do bebê em todos os sentidos. Uma mãe que não é bem vinculada talvez não vá estar muito atenta, apesar de ter sido orientada. Então, dependendo de como é construído o processo de vinculação da mãe nas orientações, vai ser o cuidado dela em casa (E2).

Se for uma mãe que não está vinculada aqui [UTIN] com o bebê, muitas vezes, em casa ela não vai conseguir esse processo de vinculação. Então, a orientação, às vezes, não é tudo. Você orienta, mas a mãe não tem condições emocionais de suprir as necessidades do bebê, por uma particularidade dela. Já tivemos bebês que a mãe não queria vê-lo porque o mesmo iria para adoção, e o bebê saiu depois da alta no colo da mãe, a mãe querendo muito o bebê. Então, a gente tenta resgatar esse processo de vinculação (E5).

Essas falas demonstram a necessidade de detectar precocemente casos de vínculos mal formados e implementar medidas para reverter esse processo, preparando a família e também o ambiente para onde o bebê irá retornar, visando desenvolver senso de responsabilidade nos pais para o cuidado ao RN. Diante dessa questão, percebe-se que 0 apego ao bebê se desenvolve em um processo contínuo de aprendizagem, no qual os pais aprendem sobre si mesmos e sobre o filho, passando a experimentar situações nas quais o bebê responde positivamente aos seus cuidados.

Por isso, é importante a consciência, por parte dos profissionais, da interferência negativa ocasionada pelo despreparo, pois, dessa forma, o enfermeiro é motivado a se preparar adequadamente para prestar uma assistência de qualidade e que favoreça a formação do vínculo e do apego entre pais e filho(a) ${ }^{16}$.

\section{Percepção dos pais sobre as orientações de cuidado ao recém-nascido}

0 processo de alta hospitalar do RN é constituído também por inúmeras orientações fornecidas aos pais pelos profissionais, em relação a vários aspectos. Todavia, a maioria dos pais entrevistados relatou não ter recebido nenhum tipo de orientação ou capacitação antes da alta de seu(sua) filho(a). Outros apontaram não entender as orientações passadas. Tal afirmativa é confirmada pelas falas:

[Não recebi informação] nenhuma. Ela [RN] ainda não tem previsão [de alta], conforme vai chegando mais perto, que eles [profissionais] observam que ela está se recuperando, então, eles chamam (P1).

[Gostaria que] a gente pudesse receber informações mais concretas. Assim poderíamos ter uma visão melhor da alta e de como eu devo proceder, de quanto em quanto tempo eu tenho que levar ele [RN] no pediatra novamente, se pode ou não se repetir o caso da doença dele. Mas eu tenho que saber durante a internação o que realmente está acontecendo, que não haja necessidade da gente estar sempre procurando e perguntando. Que o médico venha aí e nos diga: Aconteceu isso, por causa disso, daquilo e daquele outro (P9).

Por meio desses relatos percebe-se que há divergências entre o que foi falado pelos profissionais e pelos pais. Sendo assim, na percepção dos pais, os profissionais não desenvolvem ações de educação em saúde vol-tadas para o cuidado do RN em seu domicílio de modo efetivo. Com relação a esta questão, um estudo ${ }^{17}$ evidenciou que os pais não estão preparados para a alta hospitalar do(a) filho(a) e tem dúvidas quanto a isso, sendo que as percepções deles são diferentes das percepções dos profissionais de saúde.

Em virtude disso, avaliar a percepção dos pais tornase relevante por retratar, de forma indireta, os resultados da efetivação das ações de enfermagem e de outros 
profissionais da saúde, entendendo que a percepção é de-finida como o modo pelo qual o sujeito organiza e inter-preta as informações obtidas por meio das suas impressões sensoriais ${ }^{18}$.

Nesse contexto, enfatiza-se ainda a importância de considerar a forma como os profissionais repassam essas informações aos pais. Sendo assim, a fala abaixo ressalta essa questão:

Queria que ele (médico) explicasse melhor (...), não entendi direito o que ele quis dizer (P8).

Diante disso, as orientações fornecidas no processo de alta dos bebês devem ser claras, concisas e de simples entendimento, para facilitar a compreensão dos pais. Em complemento, utilizar termos adequados ao nível cultural de cada família facilita para que esta compreenda as informações da forma mais adequada possívele ${ }^{6}$.

Tais deficiências no fornecimento de informações, no tocante à ausência ou na forma como são repassadas, podem refletir no preparo dos pais em executar os cuidados, como manifestado a seguir:

Se ela [RN] ficar dependendo da gente para aspirar [...], para se alimentar, então, precisaremos de uma boa instrução. Até eu conversei com a psicóloga, caso minha filha fique com bastante sequela (P1).

[Gostaria de saber sobre] o procedimento de aspiração dela [RN], da alimentação, mas é que a gente não sabe como é para ir ajudando (P10).

A partir desses depoimentos ressalta-se a importância de prover informações consistentes e claras aos pais, bem como a necessidade do desenvolvimento de formas de comunicação entre equipe e pais que facilitem o entendimento das notícias. Então, oferecer suporte emocional nos momentos de agravamento das condições clínicas do neonato é necessário, uma vez que, para os pais, receber a notícia de regressão das condições de saúde do filho pode gerar ansiedades e angústias que não só trazem sofrimento como podem, até mesmo, comprometer o entendimento da situação relatada ${ }^{15}$.

Diante disso, é fundamental discutir questões relacionadas às necessidades de pais de crianças internadas em UTIN no tocante ao fornecimento de informações acuradas e compreensíveis sobre a situação e os cuidados que deverão prestar aos seus(suas) filhos(as) no domicílio. Todavia, cabe ressaltar que umas das limitações deste estudo é o fato de os resultados não serem generalizáveis, uma vez que trata-se de um estudo qualitativo e reflete a realidade do local escolhido como cenário de pesquisa.

\section{CONCLUSÃO}

A realização deste estudo permitiu compreender a percepção dos profissionais e pais em relação ao planejamento e efetivação da alta do RN da UTIN, e como a equipe procura desenvolver o processo de educação para alta com os pais. Assim, constatou-se que na UTIN as atividades relacionadas à determinação da alta dos neonatos têm centralidade no médico, sendo ele quem decide quando o RN dará alta da Unidade e para onde ele será encaminhado, se para Unidade Pediátrica ou domicílio. Além disso, o processo de alta está focado no restabelecimento das condições fisiológicas da criança.

Identificou-se, ainda, que a alta hospitalar dos RN é percebida pelos profissionais de maneira menos complexa do que deveria ser, sendo que a maioria das orientações de cuidado, dadas aos pais antes da alta, é feita pelos enfermeiros. Entretanto, muitas dessas orientações são feitas rapidamente em vista da demanda de serviço do local, tornando-se insuficientes para que os pais consigam incorporar e compreender o cuidado realizado ao RN prematuro dentro da perspectiva de educação em saúde.

Outro achado desse estudo foi a necessidade de manter um vínculo com os pais, para que a inserção dos mesmos na UTIN possa responder às necessidades do neonato, sendo imprescindível a construção do vínculo entre o bebê e os pais ainda no hospital, na tentativa de garantir um cuidado eficiente no domicílio. Verificou-se também que os pais apresentam dúvidas e inseguranças em relação aos cuidados básicos com o(a) filho (a), além de demonstrarem insatisfação com as orientações recebidas.

Isso requer uma reflexão por parte dos profissionais da saúde acerca da forma como estão sendo desenvolvidas suas ações educativas no processo de alta hospitalar do RN da UTIN, mas também repensar sua atuação como educador em saúde e articulador na atenção à saúde dos indivíduos nos diversos níveis de assistência, já que outros estudos realizados com esses sujeitos apontam resultados semelhantes aos obtidos com essa investigação.

É valido destacar o papel importante dos serviços de apoio no atendimento aos RN, uma vez que servem de referência na continuação da assistência prestada e formam um elo no estabelecimento do crescimento e desenvolvimento saudável do prematuro.

Portanto, faz-se necessário que os enfermeiros e demais membros da equipe de saúde ampliem as estratégias de educação com pais desses pacientes na sua rotina de trabalho, visando um melhor planejamento de alta para que haja uma continuidade do cuidado. 


\section{REFERÊNCIAS}

1 Ministério da Saúde (BR). Secretaria de Atenção à Saúde. Atenção à saúde do recém-nascido: guia para os profissionais de saúde. $4^{\mathrm{a}}$ ed. Brasilia(DF); 2011.

2 Sims DC, Jacob J, Mills MM, Fett PA, Novak G. Evaluation and development of potentially better practices to improve the discharge process in the neonatal intensive care unit. Pediatrics 2006; 118(2): 115-23.

3 Silva ND, Vieira MRR. A atuação da equipe de enfermagem na assistência ao recém-nascido de risco em um hospital de ensino. Arq Cienc Saude. $2008 \mathrm{jul} / \mathrm{set} ; 15(3):$ 110-16.

4 Santos IMM, Santos RS. A maternagem de mulheres com filho prétermo: bases para a assistência de enfermagem neonatal. Rev Pesqui Cuid Fundam. [on-line] 2010 out/dez; [citado 2012 fev 01]; 2(supl.): 692-96. Disponível em: http://www.seer.unirio.br/index.php/cuidadofundamental/ article/view/1093/pdf_259

5 Dias RAB, Almeida MFPV, Araújo GF. 0 recém-nascido portador de mielomeningocele: a construção do vínculo mãe-bebê. Rev Soc Bras Enferm Ped. [on-line]. 2007 jul; [citado 2012 fev 01]; 7(1): 17-24. Disponível em: http://www.sobep.org.br/revista/images/stories/pdfrevista/vol7-n1/v.7_n.1-art2.pesq-o-recem-nascido-portador-demielomeningocele.pdf.

6 Sousa IC, Silva LMS, Guimarães TA. Preparo para a alta hospitalar de recém-nascido em unidade de tratamento intensivo neonatal: uma visão da família. Pediatria. 2008; 30 (4): 217-27.

7 Pereira AL. Educação em saúde. In: Ensinando a cuidar em saúde pública. [Rio de Janeiro]: Difusão; 2003.

8 Rodrigues LS, et al. Seguimento de prematuros após alta hospitalar. Acta Medica. 2007; 28: 559-68.

9 Bardin L. Análise de conteúdo. São Paulo: Ed 70; 2011. 229 p.

10 Oliveira SR. A alta do recém-nascido da unidade de terapia intensiva neonatal.[dissertação] Belo Horizonte: Escola de Enfermagem, Universidade Federal de Minas Gerais; 2009.

11 Rabelo MZS, Chaves EMC, Cardoso MVLML, Sherlock MSM. Sentimentos e expectativas das mães na alta hospitalar do recém-nascido prematuro. Acta Paul Enferm. 2007; 20(3): 333-37.

12 Paschoal AS, Mantovani M, Méier MJ. Percepção da educação permanente, continuada e em service para enfermeiros de um hospital de ensino. Rev Esc Enferm USP. 2007; 41(3): 478-84.

13 Rose C, Ramsay L, Leaf A. Strategies for getting preterm infants home earlier. Arch Dis Child, London. 2008; 93(4): 271-73.

14 Molina RCM, Varela PLR, Castilho SA, Bercini LO, Marcon SS. Presença da família nas unidades de terapia intensiva pediátrica e neonatal: visão da equipe multiprofissional. Esc Anna Nery. 2007; 11(3): 437-44.
15 Vieira CS, Mello DF. 0 seguimento de saúde da criança pré-termo e de baixo peso egressa da terapia intensiva neonatal. Texto \& Contexto Enferm. $2009 \mathrm{jan} / \mathrm{mar} ; 8(1):$ 74-82.

16 Nogueira PTL, Santos IMM. 0 processo de aleitamento materno de recém-nascidos pré-termo: bases para a assistência em enfermagem. Rev Pesqui Cuid Fundam. [on-line]. 2010 out/dez; [citado 2012 fev 01]; 2(supl.): 1008-10. Disponível em: http://www.seer.unirio.br/index.php/ cuidadofundamental/article/view/897/pdf_314.

17 Sneath D. Discharge teaching in the NICU: are parents prepared? An integrative review of parents' perceptions. Neonatal Network. 2009; 28(4): 237-46.

18 Pinheiro EM, Balbino FS, Balieiro NMFG, Domenico EBL, Avena MJ. Percepções da família do recém-nascido hospitalizado sobre a comunicação de más notícias. Rev Gaucha Enferm. 2009; 30(1): 77-84.

\section{NOTA}

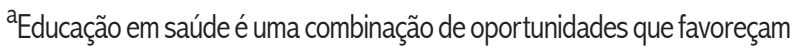
a manutenção da saúde e sua promoção, não entendida somente como transmissão de conteúdos, mas também como a adoção de práticas educativas que busquem a autonomia dos sujeitos na condução de sua vida; ou seja, nada mais é que o pleno exercício de construção da cidadania $^{7}$ 